\title{
miR-20b reduces 5-FU resistance by suppressing the ADAM9/EGFR signaling pathway in colon cancer
}

\author{
QIANG FU $^{1 *}$, JING CHENG $^{2 *}$, JINDAI ZHANG $^{1}$, YONGLEI ZHANG $^{1}$, \\ XIAOBING $\mathrm{CHEN}^{3}$, SUXIA LUO $^{3}$ and JIANGUO XIE ${ }^{1}$ \\ ${ }^{1}$ Department of Gastrointestinal Surgery Center, Henan Cancer Hospital, Zhengzhou, Henan 450008; \\ ${ }^{2}$ Department of Medical Oncology, Zhengzhou Central Hospital, Zhengzhou, Henan 450007; \\ ${ }^{3}$ Department of Digestion and Medical Oncology, Henan Cancer Hospital, Zhengzhou, Henan 450008, P.R. China
}

Received May 20, 2016; Accepted June 29, 2016

DOI: $10.3892 /$ or.2016.5259

\begin{abstract}
Chemoresistance is a major obstacle to cancer therapy including that of colon cancer (CC). Although the dysregulation of many miRNAs has been implicated in 5-fluorouracil (5-FU) resistance in CC cells, the specific role of miR-20b in chemoresistance has not been documented. In the present study, we first determined the expression of miR-20b by RT-PCR and the levels of a disintegrin and metalloprotease 9 (ADAM9) and epidermal growth factor receptor (EGFR) by western blotting in $\mathrm{CC}$ and adjacent non-cancerous tissues from 5-FU-sensitive or -resistant CC patients. Subsequently, 5-FU-sensitive (HCT116) and -resistant (HCT116-R) cells were obtained, and the levels of miR-20b, ADAM9 and EGFR were detected. Meanwhile, the 5-FU resistance of the cells was examined by assessing cell viability (by MTT assay) and apoptosis (by flow cytometry). After transfection of miR-20b into HCT116-R cells, drug resistance was reexamined. We then confirmed the relationship between miR-20b and ADAM9 by luciferase reporter assay. Finally, 5-FU resistance in HCT116 and HCT116-R cells was compared after transfection with miR-20b. Our results showed that miR-20b was expressed at lower levels in the 5-FU-resistant tissues and cells than in the 5-FU-sensitive tissues and cells. The opposite was the case for expression of ADAM9 and EGFR. In addition, we demonstrated that ADAM9 is a direct target of miR-20b and that miR-20b decreased the 5-FU resistance of HCT116-R cells. Our findings suggest that miR-20b reduces 5-FU resistance to induce apoptosis in vitro by suppressing ADAM9/EGFR in CC cells.
\end{abstract}

Correspondence to: Dr Jianguo Xie, Department of Gastrointestinal Surgery Center, Henan Cancer Hospital, 127 Dongming Road, Zhengzhou, Henan 450008, P.R. China

E-mail: jianguo_xie@126.com

*Contributed equally

Key words: colon cancer, 5-FU resistance, miR-20b, ADAM9, EGFR

\section{Introduction}

Colon cancer (CC) is a common neoplasm and presents a considerable disease burden worldwide. The incidence and mortality of CC in China has increased in recent decades (1). 5-Fluorouracil (5-FU) is a mainstream chemotherapeutic drug that is widely used in $\mathrm{CC}$ treatment $(2,3)$. The pharmacological effects of 5-FU arise from the blocking of DNA replication by interrupting the synthesis of pyrimidine thymidine. Furthermore, 5-FU induces cell cycle arrest and apoptosis (4). However, many CC patients display resistance and a high recurrence rate following chemotherapy with 5-FU. Therefore, drug resistance has been considered as one of the biggest obstacles in chemotherapy, and new strategies for enhancing the sensitivity of chemotherapy and preventing drug resistance are urgently needed.

Epidermal growth factor receptor (EGFR) is a member of tyrosine kinase receptors and has been reported to show a robust increase in levels in many types of cancer, such as non-small cell lung cancer (NSCLS), gastric cancer and CC $(5,6)$. The phosphorylation of EGFR leads to the activation of extracellular signal-regulated kinase (ERK) and the phosphatidylinositol 3-kinase (PI3K)/Ser/Thr kinase (AKT) pathways (7). These two pathways are correlated with cell migration and apoptosis. Meanwhile, EGFR contributes to drug resistance in tumor cells $(8,9)$. The human a disintegrin and metalloprotease 9 (ADAM9) is a membrane-anchored metalloprotease that is often markedly upregulated in several human carcinomas and accelerates the adherence and migration of $\mathrm{CC}$ and other tumor cells $(10,11)$. A recent study found that ADAM9 is critical for promoting cell proliferation in esophageal squamous cell carcinoma by targeting EGFR-AKT signaling (12). In fact, ADAM9 is considered as a key component in EGFR signaling and development $(13,14)$. Moreover, ADAMs have been suggested as the novel mechanisms involved in the drug resistance in human breast carcinoma MCF-7 and other cells $(15,16)$. Thus, we can speculate that ADAM9 may be able to increase drug resistance by regulating EGFR.

MicroRNAs (miRNAs) are small non-coding regulatory RNAs that are involved in temporal and tissue-specific eukaryotic gene regulation. These miRNAs pair with either full or partial complementary sequences in the $3^{\prime}$ untranslated 
regions (3'UTRs) of target mRNAs, leading to sequencespecific mRNA cleavage and/or translational repression (17). miRNAs have been virtually linked to the pathogenesis of many diseases and pharmacological mechanisms of drug therapy. Previous studies have indicated that specific targeting of miRNAs can open new avenues for cancer treatment by decreasing drug resistance and thereby improving the response to chemotherapy (18). In recent years, miR-20b has been found to be downregulated in many types of cancer tissues. In addition, miR-20b inhibited the proliferation, migration and invasion of bladder cancer EJ cells via targeting of cell cycle regulation and Sp-1-mediated MMP-2 expression (19). Moreover, miR-20b showed decreased expression in colon tumors relative to normal colon tissue (20). However, the role and mechanism of miR-20b in the drug resistance of CC cells remain unknown.

Given that a better understanding of the underlying mechanism of drug resistance could provide fresh insight into the treatment of CC, we examined the involvement of miR-20b in chemoresistance to 5-FU in CC cells using two isogenic HCT116 cell lines that are sensitive or resistant to 5-FU. Our results provide evidence that miR-20b can directly modulate chemoresistance in these cells by regulating its downstream target, the ADAM9/EGFR signaling pathway.

\section{Materials and methods}

Tissue samples and ethics statement. A total of 26 pairs of CC and adjacent non-cancerous tissues were collected from CC patients who were sensitive or resistant to 5-FU. All subjects were enrolled at the Henan Cancer Hospital and their ages ranged from 35 to 60 years. The $\mathrm{CC}$ patients were diagnosed according to the modified World Health Organization diagnostic criteria for CC by a pathologist. All tissue samples were obtained with informed consent, and the present study was approved by the Ethics Committee of Henan Cancer Hospital and was carried out in accordance with The Code of Ethics of the World Medical Association.

Cell culture. CC cell line HCT116 is sensitive to 5-FU, and was purchased from the American Type Culture Collection (ATCC; Manassas, VA, USA). The HCT116-R cell line is resistant to 5-FU and was generated by incubating HCT116 cells with increasing levels of 5-FU (Sigma-Aldrich, St. Louis, MO, USA) as previously described (21). Normal colon and CC cells were cultured in McCoy's 5A serum-free medium (Sigma, St. Louis, MO, USA) supplemented with $20 \mu \mathrm{g} / \mathrm{ml}$ insulin, $10 \mathrm{ng} / \mathrm{ml}$ epidermal growth factor and $4 \mu \mathrm{g} / \mathrm{ml}$ transferrin at $37^{\circ} \mathrm{C}$ and $5 \% \mathrm{CO}_{2}$. Resistant cell lines were maintained under constant treatment with the drug.

Cell transfection. The cells were seeded in 6-well plates at $1.0 \times 10^{5} / \mathrm{ml} /$ well and transfected in logarithmic growth phase with $25 \mathrm{nmol} / \mathrm{l} \mathrm{miR-20b}$ mimic, miR-20b inhibitor or its nonspecific control (NC). All transfection reagents were purchased from GenePharma Co., Ltd. (Shanghai, China). Transfection was performed according to the manual provided with the siPOR $^{\mathrm{TM}}$ NeoFX $^{\mathrm{TM}}$ Transfection Agent (Ambion, Grand Island, NE, USA) and the transfection efficiency was determined by RT-PCR. Aliquots of $0.5 \mu \mathrm{g}$ pcDNA3.1/ADAM9 and
pcDNA3.1 control vector were obtained from OriGene and

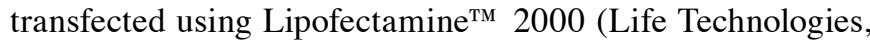
Carlsbad, CA, USA) as per the manufacturer's protocol.

Cell proliferation and cell viability assays. Cells were plated in 96-well plates at a concentration of $2 \times 10^{4}$ cells/100 $\mu \mathrm{l}$ and treated with 5 -FU $(0,5$ and $10 \mu \mathrm{g} / \mathrm{ml}$, respectively) for the indicated time to assess proliferation. The surviving fractions were stained for $2 \mathrm{~h}$ at 1-5 days with thiazolyl blue tetrazolium bromide (MTT) (Sigma). The absorbance at $570 \mathrm{~nm}$ was measured by a spectrophotometer after dissolving in dimethyl sulfoxide (DMSO) (Sigma). Cell viability was calculated as the ratio of optical density (OD) values according to the manufacturer's instructions using a CellTiter 96 AQueous One Solution Cell Proliferation Assay kit (Promega Corporation, Madison, WI, USA).

Cell cycle analysis. Cells were seeded into 6-well plates at $5 \times 10^{5}$ cells/well. After pretreatment with serum-free medium for $48 \mathrm{~h}$, the cells were harvested and fixed overnight with ice-cold $70 \%$ ethanol. The fixed cells were quantified using a Cell Cycle Analysis kit (KeyGen Biotech, Nanjing, China) according to the manufacturer's instructions. Each experiment was performed in triplicate.

Apoptosis assay. After transfection for $72 \mathrm{~h}$, the cells were collected and incubated in phosphate-buffered saline (PBS) for $10 \mathrm{~min}$. Analysis of apoptosis was performed using an Annexin $\mathrm{V}$ apoptosis detection kit (BD Pharmingen ${ }^{\mathrm{TM}}$, San Diego, CA, USA) according to the manufacturer's instructions. Cells were washed and suspended in $100 \mathrm{ml}$ binding buffer, at a density of $1 \times 10^{6}$ cells $/ \mathrm{ml}$, and then labeled with $5 \mu \mathrm{l}$ of Annexin V-FITC and PI for $15 \mathrm{~min}$. Thereafter, flow cytometric analysis was performed on a FACSAria flow cytometry system (BD Biosciences). Data were analyzed using BD FACSDiva software.

Luciferase reporter assay. The pGL3 control vector (Promega) carrying the 3'UTR sequence of ADAM9, which was predicted to interact with miR-20b or a mutated sequence within the predicted target sites, was transfected into HCT116-R cells. Before luciferase activity analysis, cells were further transfected with the miR-20b mimic. Cells were divided into five groups: control, miR-20b mimic, miR-20b + pcDNA3.1, miR-20b + pcDNA3.1/ADAM9 and mimic NC. Firefly luciferase activity was measured using the Dual-Luciferase Assay kit (Promega) according to the manufacturer's instructions, and the data were then normalized using a Renilla luciferase reference plasmid.

Real-time quantitative polymerase chain reaction ( $R T$ - $q P C R)$ assay. Total RNA was extracted using TRIzol reagent (Invitrogen Life Technologies, Carlsbad, CA, USA) from cells or tissues. The concentration and integrity of the RNA were determined by electrophoresis, and the RNA was reverse transcribed using a RevertAid First Strand cDNA Synthesis kit (Invitrogen). For transfection with ADAM9, the cDNA of ADAM9 was synthesized using a TaqMan MicroRNA Reverse Transcription kit (Applied Biosystems, Foster City, CA, USA). The Maxima SYBR-Green Master Mix (2X) was 


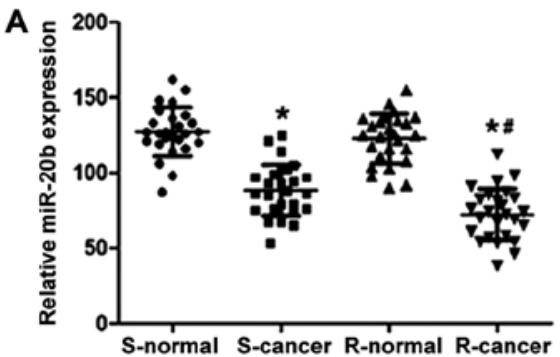

C

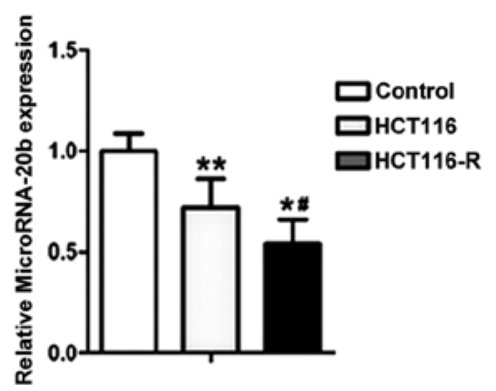

B

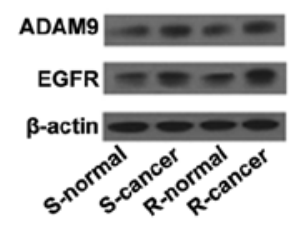

D

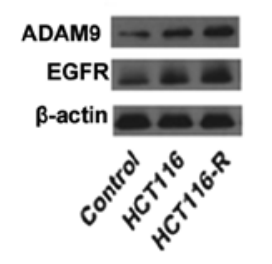

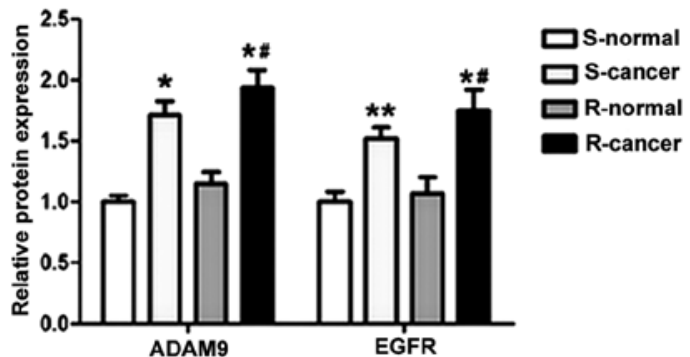

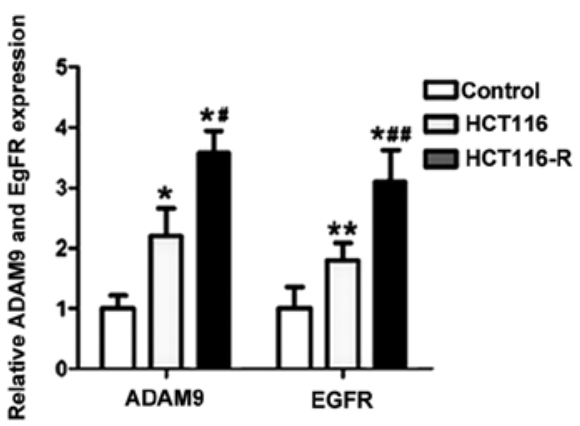

Figure 1. miR-20b is downregulated, whereas ADAM9 and EGFR are upregulated in CC patient tissues and CC cells that are resistant to 5-FU. (A) The level of miR-20b was assessed by RT-PCR in CC tissues and their matched adjacent non-cancerous tissues. Data are presented as mean \pm SD ( $=26)$; ${ }^{*}<0.01$ vs. the matched adjacent non-cancerous tissues; ${ }^{~} \mathrm{P}<0.01$ vs. sensitive (S-cancer) group. (B) The expression of ADAM9 and EGFR was measured by western blotting in CC tissues and their matched adjacent non-cancerous tissues. Data are presented as mean $\pm \mathrm{SD}(\mathrm{n}=4)$; ${ }^{*} \mathrm{P}<0.01$ and ${ }^{* *} \mathrm{P}<0.001$ vs. the matched adjacent non-cancerous tissues; ${ }^{\prime} \mathrm{P}<0.01$ vs. S-cancer group. (C) The level of miR-20b in normal colon cells and HCT116 and HCT116-R cell lines. (D) The level of ADAM9 and EGFR in normal colon cells and HCT116 and HCT116-R cell lines; ${ }^{*} \mathrm{P}<0.01$ and ${ }^{* *} \mathrm{P}<0.001$ vs. control group; ${ }^{*} \mathrm{P}<0.01$ and ${ }^{\# \#} \mathrm{P}<0.001$ vs. HCT-116 group. R, resistant; $\mathrm{S}$, sensitive.

obtained from Applied Biosystems according to the manufacturer's instructions. The PCR involved an initial step at $95^{\circ} \mathrm{C}$ for $10 \mathrm{~min}$, followed by 40 cycles of $95^{\circ} \mathrm{C}$ for $15 \mathrm{sec}$, $60^{\circ} \mathrm{C}$ for $1 \mathrm{~min}$ and $72^{\circ} \mathrm{C}$ for $45 \mathrm{sec}$. Primer pairs for miR-20b were designed and synthesized by RiboBio Inc. (Guangzhou, China). Data were analyzed using the comparative $2^{-\Delta \Delta C t}$ method.

Western blotting. Cells were homogenized with lysate (Beyotime) supplemented with $1 \mathrm{mM}$ phenylmethylsulfonyl fluoride (PMSF) (Sigma), and then centrifuged. The supernatants were collected and the contents were quantified using the BCA kit (Pierce, Rockford, IL, USA). Samples of $20 \mu \mathrm{g}$ protein were separated by SDS-PAGE using a $12 \%$ polyacrylamide gel and transferred to a polyvinylidene difluoride (PVDF) membrane (Millipore, Billerica, MA, USA). After blockage of non-specific binding sites with $5 \%$ non-fat milk, the membrane was incubated overnight at $4^{\circ} \mathrm{C}$ with various primary antibodies, including antibodies against ADAM9, EGFR and $\beta$-actin [Cell Signaling Technology (CST); Danvers, MA, USA)] (1:500). Next, the nitrocellulose membrane was washed three times with PBS containing $0.1 \%$ Tween-20 and further incubated with the secondary antibody (1:800) at room temperature. The immunoblots were visualized using an ECL Western Blotting Detection kit (Millipore) and analysis software Quantity One (Bio-Rad, Hercules, CA, USA).

Statistical analysis. Quantitative data are expressed as mean \pm standard deviation (SD). Measurement data were analyzed using Student's t-test when there were only two groups. For other situations, statistical analyses were performed using one-way or two-way ANOVA in SPSS 19.0. The level of significance was set at $\mathrm{P}<0.01$.

\section{Results}

miR-20b is downregulated, while ADAM9 and EGFR are upregulated in tissues of the 5-FU-resistant CC patients. To investigate the potential roles of miR-20b in CC, we first examined the expression of miR-20b in an expanded cohort of $26 \mathrm{CC}$ patients. Our results showed that miR-20b expression was markedly downregulated in the CC tissues compared to the level in the corresponding adjacent non-cancerous tissues (Fig. 1A). Meanwhile, miR-20b expression was lower in the $\mathrm{CC}$ patients who were sensitive to 5-FU than the level in those who were resistant. In order to identify the role of ADAM9 and EGFR in 5-FU resistance, the levels of ADAM9 and EGFR were detected by western blotting. As shown in Fig. 1B, the expression levels of ADAM9 and EGFR were significantly higher in the cancer cells than levels in the normal control cells. Similarly, levels of ADAM9 and EGFR expression were higher in $\mathrm{CC}$ patients who were sensitive to 5-FU than these levels noted in the patients who were resistant.

To further explore the mechanisms of 5-FU resistance in the two isogenic CC cell lines (HCT116 and HCT116-R), HCT116, HCT116-R and normal CC cells were cultured and the levels of miR-20b (Fig. 1C), ADAM9 and EGFR (Fig. 1D) were measured. The results for miR-20b, ADAM9 and EGFR expression in the $\mathrm{CC}$ tissue and $\mathrm{CC}$ cell lines were consistent. These data showed that the expression of miR-20b was universally lower whereas levels of ADAM9 and EGFR were higher in the 5-FU resistant CC patients 
A

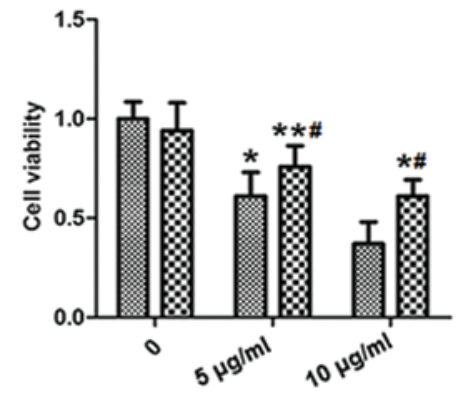

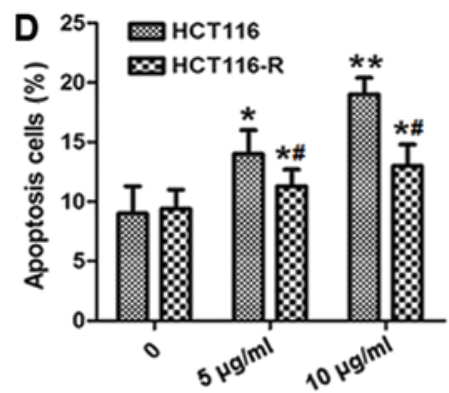

B

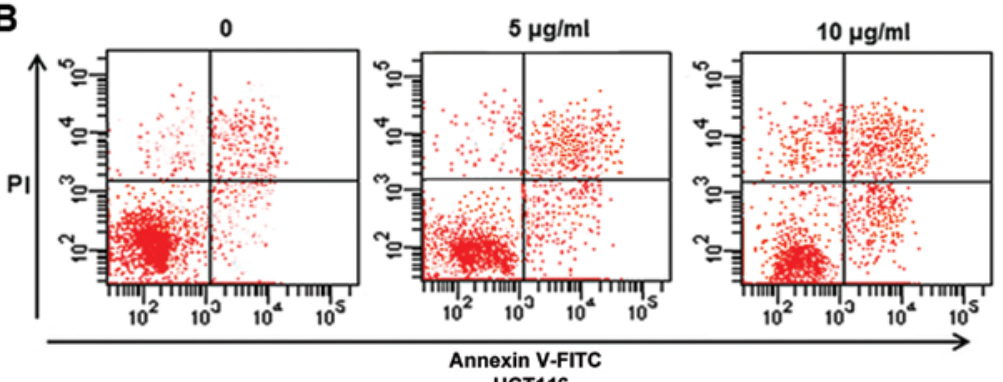

HCT116

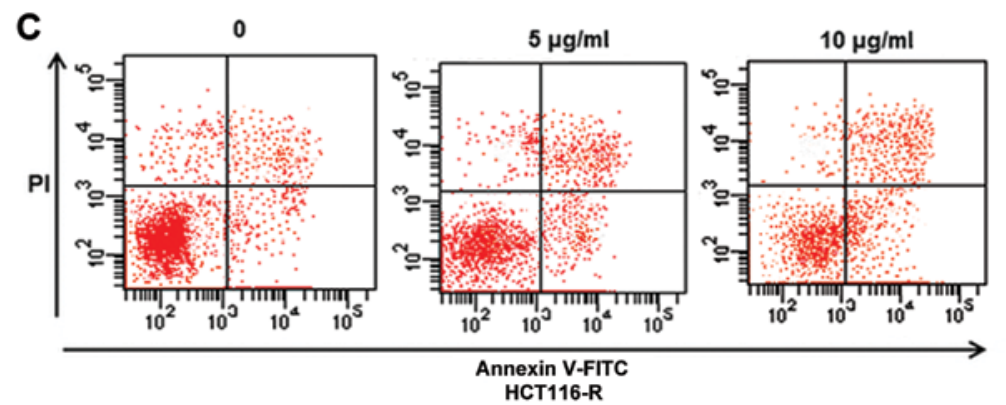

Figure 2. HCT116-R cell line is resistant to 5-FU. HCT116 and HCT116-R cells were treated with 0,5 or $10 \mu \mathrm{g} / \mathrm{ml} 5$-FU for $72 \mathrm{~h}$ and were then used for study. (A) Cell viability was detected by MTT assay. (B and C) Cell apoptosis in HCT116 and HCT116-R cells was analyzed by flow cytometry. (D) Quantification of relative numbers of apoptotic cells. Data are presented as mean $\pm \mathrm{SD}(\mathrm{n}=4) ;{ }^{*} \mathrm{P}<0.01$ and ${ }^{* * *} \mathrm{P}<0.001$ vs. the untreated group; ${ }^{*} \mathrm{P}<0.01 \mathrm{vs}$. the parental HCT116 group.

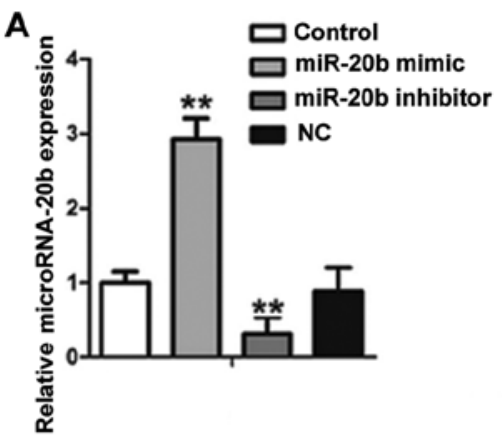

D

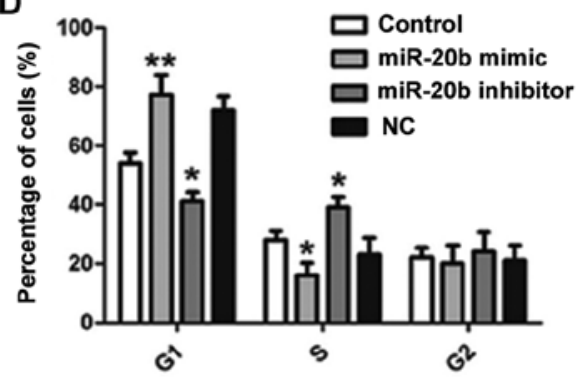

B

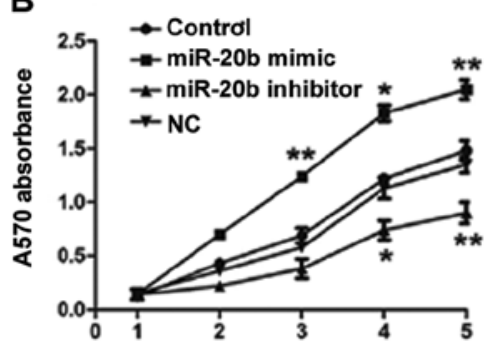

E
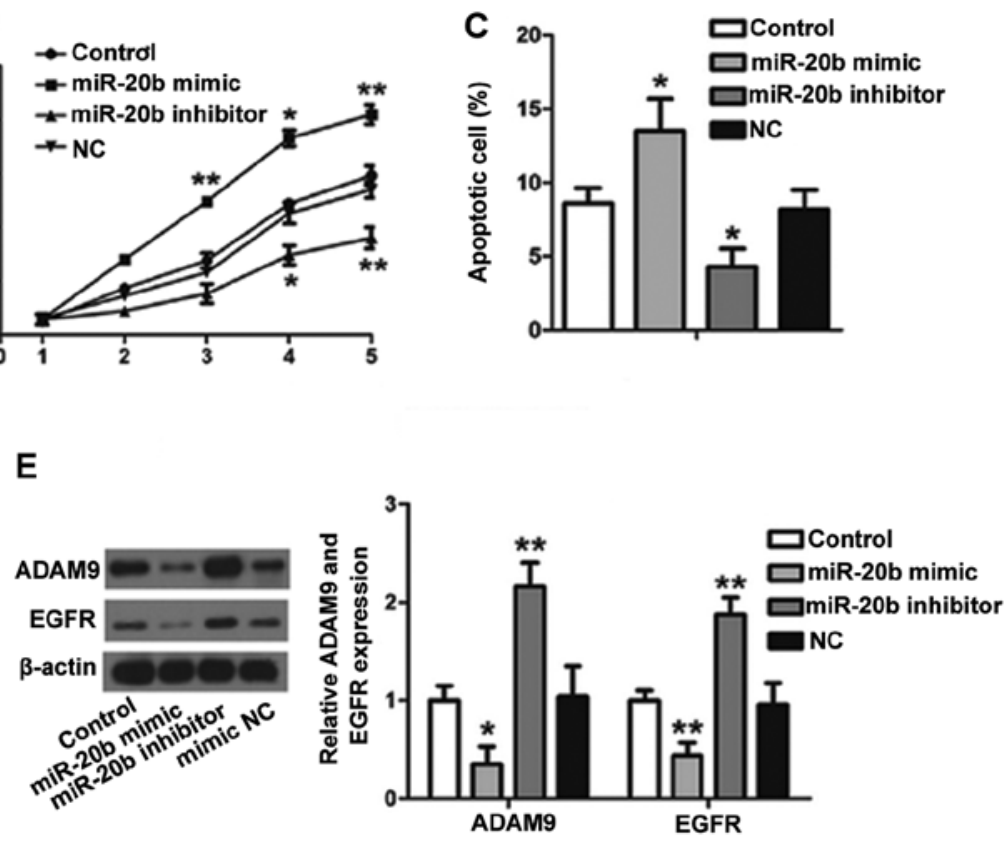

Figure 3. miR-20b inhibits proliferation and induces apoptosis in HCT116-R cells. HCT116-R cells were transfected with miR-20b and were then used for study. (A) The level of miR-20b in HCT116-R cells among the four groups. (B) Proliferation rate of HCT116-R cells was detected by the MTT assay in the four groups. (C) Quantification of relative numbers of apoptotic cells in the four groups. (D) Cell cycle analyses in HCT116-R cells in the four groups. (E) Protein fold-change of ADAM9 and EGFR in HCT116-R cells in the four groups. Data are presented as mean $\pm \mathrm{SD}(\mathrm{n}=4)$; ${ }^{*} \mathrm{P}<0.01$ and ${ }^{* *} \mathrm{P}<0.001$ vs. control group. 
A

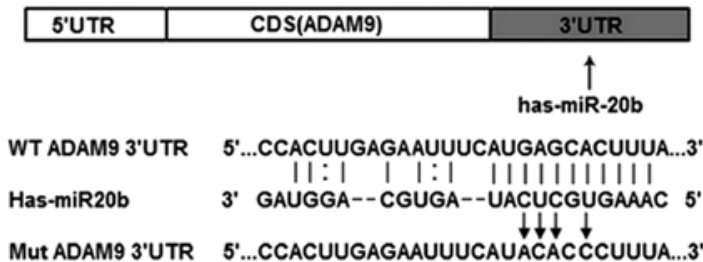

B

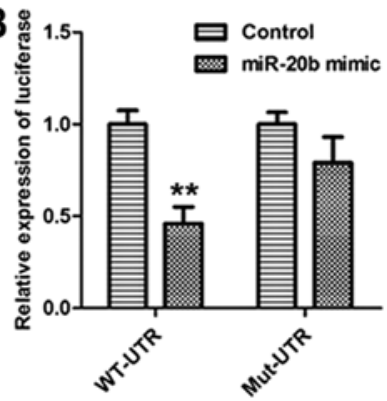

C

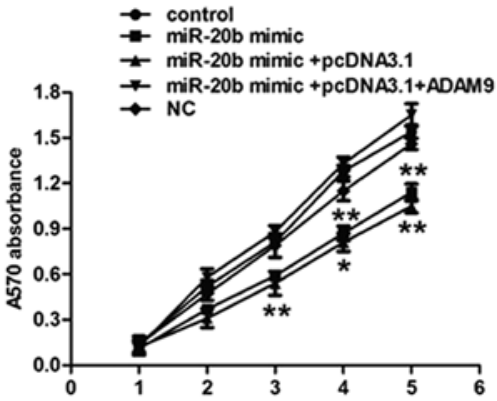

E

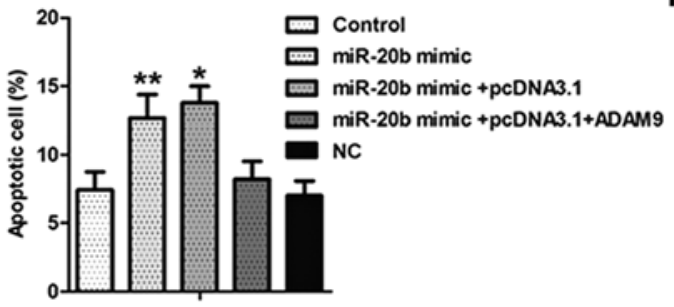

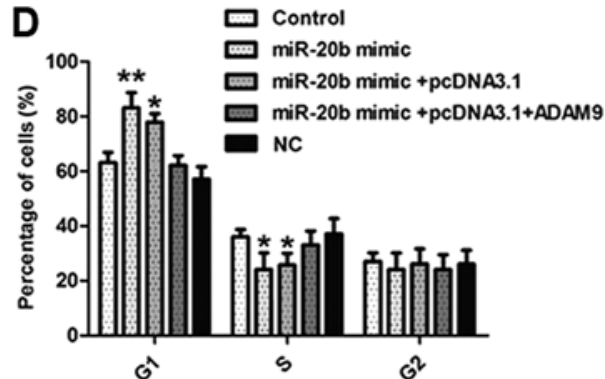
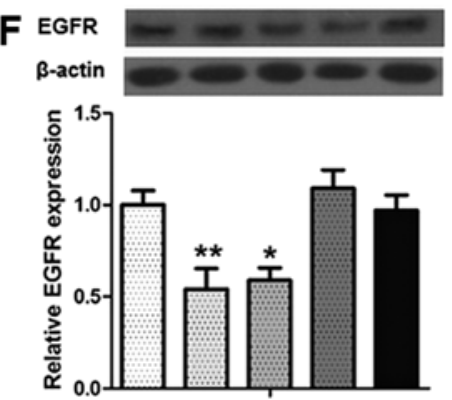

Figure 4. miR-20b suppresses cell proliferation and apoptosis and regulates cell cycle progression by targeting ADAM9 in HCT116-R cells. (A) The wild-type 3'UTR of human ADAM9 mRNA contains a putative miR-20b-binding site. The mutant form is shown. (B) Relative luciferase activity of HCT116-R cells cotransfected with miR-20b mimic and ADAM9 3'UTR-luciferase reporter vector which contained the wild-type sequence (ADAM9-3'UTR-wt), or in a vector which contained a mutant sequence (ADAM9-3'UTR-mut) within the control. (C) HCT116-R cells were co-transfected with miR-20b and pcDNA3.1/ADAM9, and then the proliferation rate of HCT116-R cells was detected using MTT assay. (D) Cell cycle analyses in HCT116-R cells were conducted after cotransfection. (E) HCT116-R cells were co-transfected with miR-20b and pcDNA3.1/ADAM9 and the apoptotic rates were determined. (F) Protein expression of EGFR in HCT116-R cells was detected after co-transfection. Data are presented as mean $\pm \mathrm{SD}(\mathrm{n}=4)$; ${ }^{*} \mathrm{P}<0.01$ and ${ }^{* *} \mathrm{P}<0.001$ vs. control group.

and cells than in the resistant patients and cells, indicating that miR-20b, ADAM9 and EGFR may be involved in 5-FU resistance in CC cells.

HCT116-R cell line is resistant to 5-FU. To understand the mechanisms of 5-FU resistance, we determined the effect of 5-FU on the growth of HCT116 and HCT116-R cell lines, and found that 5-FU decreased cell viability in a dose-dependent manner, with a more obvious effect on HCT116 cells than HCT116-R cells (Fig. 2A). Fig. 2B and C respectively show the percentage of apoptosis in the HCT116 and HCT116-R cells after 5-FU treatment. The statistical data on the percentage of apoptotic cells is shown in Fig. 2D. Similarly, HCT116 cells showed higher sensitivity to 5-FU-induced apoptosis than HCT116-R cells, as demonstrated by cell viability.

miR-20b mediates proliferation and apoptosis in HCT116-R cells in vitro. To identify the role of miR-20b in CC cells, miR-20b was overexpressed or suppressed in the HCT116-R cells by transfection with miR-20b mimic or miR-20b inhibitor (Fig. 3A). As shown in Fig. 3B, cell proliferation was decreased in the miR-20b-overexpressed group in comparison with the control group according to the MTT assay. Conversely, in the miR-20b-suppressed group, cell proliferation was accelerated. Moreover, the percentage of apoptotic cells increased to $13.5 \%$ after transfection with the miR-20b mimic (Fig. 3C). Inhibition of the expression of miR-20b decreased the apoptosis of the HCT116-R cells.

Next, we sought to determine whether miR-20b has any impact on cell cycle progression in the HCT116-R cells (Fig. 3D). We found that the number of cells in the G1 phase was increased in the miR-20b mimic group compared with this percentage in the control, but this percentage was decreased in the $\mathrm{S}$ phase. Meanwhile, the miR-20b mimic and miR-20b inhibitor groups showed contrasting results. The percentage of cells in G2 did not differ significantly between groups. In addition, the protein expression of ADAM9 and EGFR was downregulated in the miR-20b mimic group and upregulated in the miR-20b inhibitor group compared with the control (Fig. 3E). Overall, these findings suggest that miR-20b inhibits the proliferation and induces the apoptosis of $\mathrm{CC}$ by suppressing cell cycle progression at the G1/S transition in CC 
A
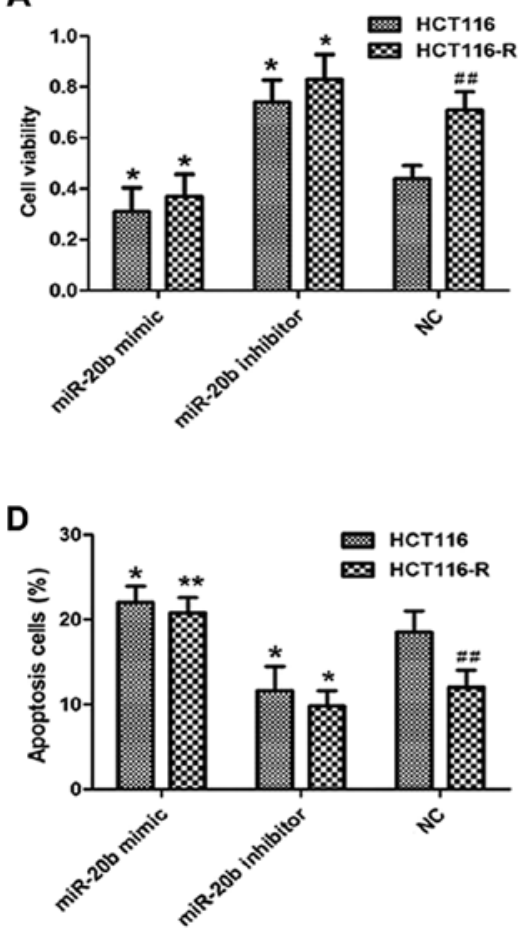

B

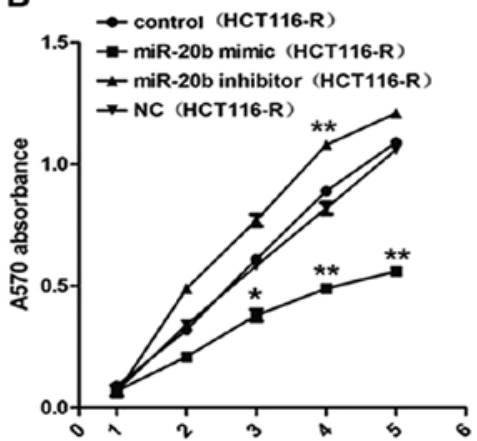

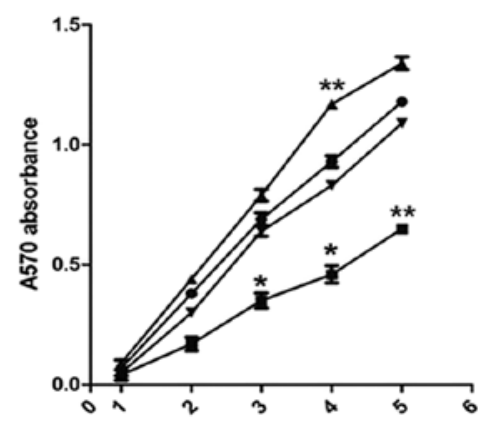

$\mathbf{F}$

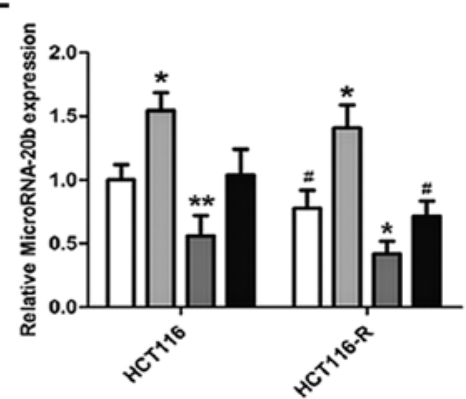

C

G

E

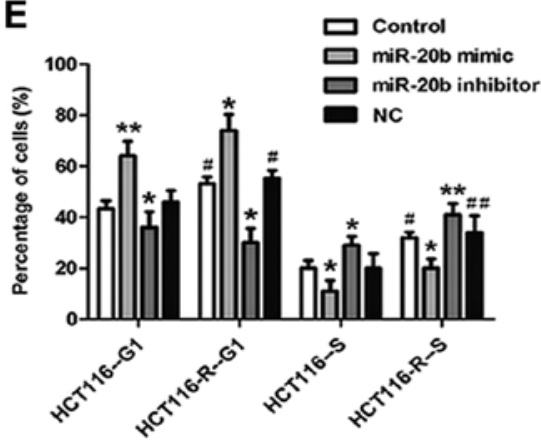

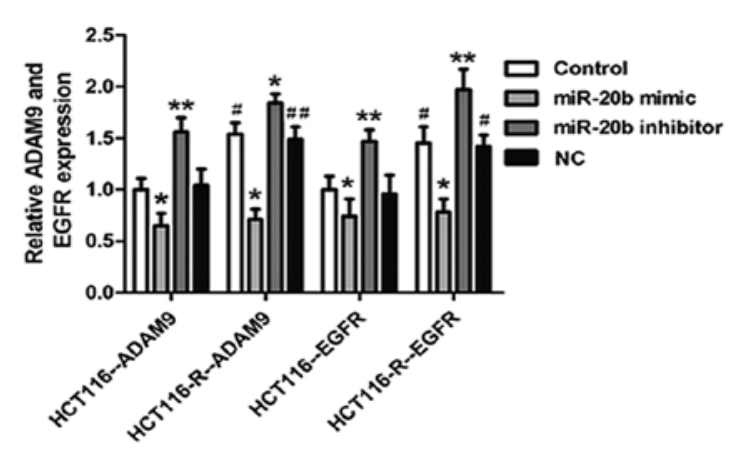

Figure 5. Detection of drug resistance to 5-FU in HCT116 and HCT116-R cells after transfection with miR-20b. HCT116 and HCT116-R cells were transfected with miR-20b and then treated with $10 \mu \mathrm{g} / \mathrm{ml} 5$-FU for $72 \mathrm{~h}$. All cells were collected and used for further study. (A) Cell viability in HCT116 and HCT116-R cells; ${ }^{2} \mathrm{P}<0.01$ vs. their own NC group; ${ }^{\# \#} \mathrm{P}<0.001$ vs. their parental HCT116 group. (B and C) Proliferation rates of HCT116 and HCT116-R cells. (D) Relative numbers of apoptotic cells in HCT116 and HCT116-R cells; ${ }^{*} \mathrm{P}<0.01$ and ${ }^{* *} \mathrm{P}<0.001$ vs. their own NC group; ${ }^{\# \#} \mathrm{P}<0.001$ vs. their parental HCT116 group. (E) Cell cycle analyses in HCT116 and HCT116-R cells. (F) The level of miR-20b in HCT116 and HCT116-R cells. (G) Protein fold-change of ADAM9 and EGFR in HCT116 and HCT116-R cells. Data are presented as mean $\pm \mathrm{SD}(\mathrm{n}=4) ;{ }^{*} \mathrm{P}<0.01$ and ${ }^{* *} \mathrm{P}<0.001$ vs. their own control group; ${ }^{\sharp} \mathrm{P}<0.01$ and ${ }^{\# \#} \mathrm{P}<0.001$ vs. their parental HCT116 group.

cells. Moreover, ADAM9 and EGFR may be involved in this process.

miR-20b suppresses cell proliferation and apoptosis and regulates cell cycle progression by targeting ADAM9 in HCT116-R cells. In order to investigate the relationship between miR-20b and ADAM9/EGFR in CC, ADAM9 was identified as one of the putative targets of miR-20b. We constructed the wild-type and mutant ADAM9 and cloned it downstream of a luciferase reporter gene (Fig. 4A). We found that miR-20b decreased the luciferase intensity by $\sim 63 \%$ in the wild-type ADAM9 3'UTR of HCT116-R cells compared with the normal cells. However, luciferase activity did not differ from that in the ADAM9 3'UTR-mut groups (Fig. 4B). These results suggest that ADAM9 is a direct target of miR-20b in CC.
To further confirm that ADAM9 is a functional target of miR-20b in CC cells, we co-transfected HCT116-R cells with pcDNA3.1-ADAM9 and miR-20b mimic to construct a ADAM9 overexpression model. Our results showed an increase in cell proliferation, a reduction in cell cycle arrest at G1/S and a reduction in apoptosis rate in the ADAM9-overexpressed group (Fig. 4C-E). Accordingly, ADAM9 overexpression elevated the expression of EGFR (Fig. 4F). All these data indicate that miR-20b suppresses CC cell proliferation, apoptosis and cell cycle progression by directly targeting ADAM9.

miR-20b reduces 5-FU resistance in the HCT116-R cells. To determine whether miR-20b reduces the 5-FU resistance of CC cells, HCT116 and HCT116-R cells were transfected with miR-20b mimic. Next, these cells were treated with 
$10 \mu \mathrm{g} / \mathrm{ml} 5$-FU for $72 \mathrm{~h}$. We then assessed the cell viability, proliferation, apoptosis, cell cycle progression and expression of ADAM9 and EGFR as mentioned above. Our results showed that miR-20b suppressed cell viability and proliferation in both the HCT116 and HCT116-R cells (Fig. 5A-C). In contrast, apoptosis, cell cycle progression and ADAM9 and EGFR expression were promoted by miR-20b in the HCT116 and HCT116-R cells (Fig. 5D-G). Meanwhile, none of the measured parameters differed between HCT116 and HCT116-R cells in the mimic and miR-20b inhibitor groups. These results indicate that miR-20b attenuated the 5-FU resistance of HCT116-R cells.

Taken together, the present study demonstrated that miR-20b reduced 5-FU resistance by suppressing the ADAM9/EGFR signaling pathway in $\mathrm{CC}$ tissues and in vitro.

\section{Discussion}

5-FU is widely considered to be the standard therapy for CC and is usually combined with leucovorin. Although 5-FU can improve the median survival of $\mathrm{CC}$ patients by almost two years (22), drug resistance to 5-FU is a serious issue. In the present study, in order to investigate the potential mechanism of chemoresistance to 5-FU in CC cells, we established a 5-FU-resistant cell line from human CC cell line HCT116 and constructed an ADAM9 overexpression model by co-transfecting HCT116-R cells with pcDNA3.1-ADAM9 and miR-20b mimic. The major finding was that miR-20b was positively associated with 5-FU resistance but inversely associated with the expression of ADAM9/EGFR in CC cells. Moreover, miR-20b reduced the 5-FU resistance of $\mathrm{CC}$ cells by inhibiting ADAM9 expression, and EGFR expression was inhibited as well. These results suggest that targeting miR-20b may represent a novel therapeutic approach for CC.

Increasing evidence suggests that miRNAs play important roles in cancers and many are aberrantly expressed in $\mathrm{CC}$ cells (23-25). Ahmed proposed that miRNAs could be markers for the diagnostic screening of CC (26). In the present study, we found that miR-20b was downregulated in $\mathrm{CC}$ patients and significantly inhibited the proliferation of CC cells in vitro, which is consistent with a previous study that found that miR-20b was downregulated in colon tumors. Furthermore, miR-20b was reported to inhibit the proliferation of melanoma cells by regulating the proteinase-activated receptor-1 (PAR1), thrombin receptor (27), and to inhibit cell cycle progression by targeting MMP-2 in bladder cancer EJ cells (19).

Recent evidence has shown that miRNAs attenuate or contribute to 5-FU resistance in human CC cells (28). MicroRNA array showed that microRNA-195 chemosensitizes CC cells to the chemotherapeutic drug doxorubicin by targeting the first binding site of B-cell CLL/lymphoma 2-like protein 2 (BCL2L2) mRNA (29). Mussnich et al reported that miR-199a-5p and miR-375 affect CC cell sensitivity to cetuximab by targeting the $\mathrm{PH}$ domain and leucine rich repeat protein phosphatase 1 (PHLPP1) (30). MicroRNA-425-5p was also reported to reduce 5-FU resistance in colorectal cancer cells via the regulation of programmed cell death 10 (21). Our present study found that miR-20b reduced 5-FU resistance in $\mathrm{CC}$ cells, which may be a novel potential mechanism for suppressing 5-FU resistance in CC cells.
EGFR has been identified as a tumor-promoting gene in many types of cancers. EGFR-targeted therapy has recently been implemented as a new therapeutic strategy in various malignancies (31). The anti-EGFR monoclonal antibodies cetuximab and panitumumab showed good clinical activity in $\sim 10 \%$ of patients with metastatic colorectal cancer that was resistant to chemotherapy (32). Patel et al demonstrated that curcumin enhanced the effects of 5-FU and oxaliplatin in mediating growth inhibition of CC cells by regulating EGFR (33), which indicates that EGFR is closely linked to 5-FU resistance in CC cells. Indeed, EGFR is widely considered as a drug resistance gene in NSCLS, glioblastoma, colorectal cancer and other cancers, and could be modulated by many genes $(9,34)$. It was also found that overexpression of ADAM9 enhanced growth factor-mediated recycling of E-cadherin in the human CC cell line HT29 (35). In the present study, we demonstrated that EGFR was not only upregulated with ADAM9 synchronously in CC, but was also positively regulated by ADAM9. Furthermore, cell viability, cell cycle progression and cell proliferation were inhibited when ADAM9/EGFR were elevated, whereas cell apoptosis was accelerated. Additionally, the expression of ADAM9 and EGFR was higher in HCT116 cells than that in HCT11-R cells. Thus ADAM9/EGFR showed a positive correlation with the 5-FU resistance of CC cells. These results are consistent with previous studies that found that ADAM9 is correlated with cell adherence and migration in pancreatic cancer cells (36), and that the expression of EGFR and ADAM9 was increased and that EGFR is the downstream target gene of ADAM9 in esophageal squamous cell carcinoma cells (12). Our results thus confirm that the ADAM9/EGFR pathway plays an important role in the 5-FU resistance of CC cells.

Although ADAM9 has been reported to be upregulated in $\mathrm{CC}$, there is little research on the mechanism of action of ADAM9 in CC. The present study found that ADAM9/EGFR could be downregulated by miR-20b mimic and upregulated by miR-20b inhibitor in CC cells, supporting the hypothesis that ADAM9/EGFR is the functional downstream target of miR-20b in vitro. A luciferase reporter assay further confirmed that ADAM9 functions as a direct target of miR-20b and prevented the cell apoptosis induced by miR-20b in CC. Notably, we observed that the level of ADAM9/EGFR and 5-FU resistance did not differ between HCT11-R and HCT116 cells after transfection with miR-20b in the presence of 5-FU. These results indicate that $\mathrm{miR}-20 \mathrm{~b}$ attenuates 5-FU resistance in CC cells by depressing ADAM9/EGFR expression.

In summary, the present data suggest that miR-20b is downregulated in 5-FU-resistant CC patients and cells compared with sensitive patients and cells. 5-FU sensitivity could be increased by miR-20b through regulation of the ADAM9/EGFR pathway. Our study broadens our understanding of the contribution of miR-20b to 5-FU sensitivity in $\mathrm{CC}$ cells via the targeting of several other genes, and indicates that it could be a powerful target to reduce 5-FU resistance in CC cells.

\section{References}

1. Hou L, Ji BT, Blair A, Dai Q, Gao YT and Chow WH: Commuting physical activity and risk of colon cancer in Shanghai, China. Am J Epidemiol 160: 860-867, 2004. 
2. Li J, Hou N, Faried A, Tsutsumi S, Takeuchi T and Kuwano H: Inhibition of autophagy by 3-MA enhances the effect of 5-FU-induced apoptosis in colon cancer cells. Ann Surg Oncol 16: 761-771, 2009.

3. Longley DB, Harkin DP and Johnston PG: 5-fluorouracil: Mechanisms of action and clinical strategies. Nat Rev Cancer 3: 330-338, 2003

4. Zhang Y, Talmon G and Wang J: MicroRNA-587 antagonizes 5-FU-induced apoptosis and confers drug resistance by regulating PPP2R1B expression in colorectal cancer. Cell Death Dis 6: e1845, 2015.

5. Laurent-Puig P, Cayre A, Manceau G, Buc E, Bachet JB, Lecomte T, Rougier P, Lievre A, Landi B, Boige V, et al: Analysis of PTEN, BRAF, and EGFR status in determining benefit from cetuximab therapy in wild-type $K R A S$ metastatic colon cancer. J Clin Oncol 27: 5924-5930, 2009.

6. Pirker R, Pereira JR, von Pawel J, Krzakowski M, Ramlau R, Park K, de Marinis F, Eberhardt WE, Paz-Ares L, Störkel S, et al: EGFR expression as a predictor of survival for first-line chemotherapy plus cetuximab in patients with advanced non-small-cell lung cancer: Analysis of data from the phase 3 FLEX study. Lancet Oncol 13: 33-42, 2012.

7. Noordhuis MG, Eijsink JJH, Ten Hoor KA, Roossink F, Hollema H, Arts HJ, Pras E, Maduro JH, Reyners AK, de Bock GH, et al: Expression of epidermal growth factor receptor (EGFR) and activated EGFR predict poor response to (chemo)radiation and survival in cervical cancer. Clin Cancer Res 15: 7389-7397, 2009.

8. Zhao Y, Tan B, Kuo MS, Liu L and Breyer MD: Abstract 5439: Metabolomic study of EGFR drug resistance mechanisms. Cancer Res 74 (Suppl 19): S5439-S5439, 2014.

9. Taylor TE, Furnari FB and Cavenee WK: Targeting EGFR for treatment of glioblastoma: Molecular basis to overcome resistance. Curr Cancer Drug Targets 12: 197-209, 2012.

10. Li J, Ji Z, Qiao C, Qi Y and Shi W: Overexpression of ADAM9 promotes colon cancer cells invasion. J Invest Surg 26: 127-133, 2013.

11. Jia AY, Castillo-Martin M, Bonal DM, Sánchez-Carbayo M, Silva JM and Cordon-Cardo C: MicroRNA-126 inhibits invasion in bladder cancer via regulation of ADAM9. Br J Cancer 110 2945-2954, 2014

12. Liu R, Gu J, Jiang P, Zheng Y, Liu X, Jiang X, Huang E, Xiong S, $\mathrm{Xu}$ F, Liu G, et al: DNMT1-microRNA126 epigenetic circuit contributes to esophageal squamous cell carcinoma growth via ADAM9-EGFR-AKT signaling. Clin Cancer Res 21: 854-863, 2015.

13. Blobel CP: ADAMs: Key components in EGFR signalling and development. Nat Rev Mol Cell Biol 6: 32-43, 2005.

14. Pham NA, Schwock J, Iakovlev V, Pond G, Hedley DW and Tsao MS: Immunohistochemical analysis of changes in signaling pathway activation downstream of growth factor receptors in pancreatic duct cell carcinogenesis. BMC Cancer 8: 43, 2008.

15. Isseri OD, Kars MD, Arpaci F and Gündüz U: Gene expression analysis of drug-resistant MCF-7 cells: Implications for relation to extracellular matrix proteins. Cancer Chemother Pharmacol 65 447-455, 2010

16. Kyula JN, Van Schaeybroeck S, Doherty J, Fenning CS, Longley DB and Johnston PG: Chemotherapy-induced activation of ADAM-17: A novel mechanism of drug resistance in colorectal cancer. Clin Cancer Res 16: 3378-3389, 2010

17. Bartel DP: MicroRNAs: Target recognition and regulatory functions. Cell 136: 215-233, 2009.

18. Sarkar FH, Li Y, Wang Z, Kong D and Ali S: Implication of microRNAs in drug resistance for designing novel cancer therapy. Drug Resist Updat 13: 57-66, 2010.

19. Park SL, Cho TM, Won SY, Song JH, Noh DH, Kim WJ and Moon SK: MicroRNA-20b inhibits the proliferation, migration and invasion of bladder cancer EJ cells via the targeting of cell cycle regulation and Sp-1-mediated MMP-2 expression. Oncol Rep 34: 1605-1612, 2015.

20. Sarver AL, French AJ, Borralho PM, Thayanithy V, Oberg AL, Silverstein KA, Morlan BW, Riska SM, Boardman LA, Cunningham JM, et al: Human colon cancer profiles show differential microRNA expression depending on mismatch repair status and are characteristic of undifferentiated proliferative states. BMC Cancer 9: 401-401, 2009.
21. Zhang Y, Hu X, Miao X, Zhu K, Cui S, Meng Q, Sun J and Wang T: MicroRNA-425-5p regulates chemoresistance in colorectal cancer cells via regulation of Programmed Cell Death 10. J Cell Mol Med 20: 360-369, 2016

22. Giacchetti S, Itzhaki M, Gruia G, Adam R, Zidani R, Kunstlinger F, Brienza S, Alafaci E, Bertheault-Cvitkovic F, Jasmin C, et al: Long-term survival of patients with unresectable colorectal cancer liver metastases following infusional chemotherapy with 5-fluorouracil, leucovorin, oxaliplatin and surgery. Ann Oncol 10: 663-669, 1999.

23. Linda Y, Narasimhaswamy B and David HB: MicroRNA and colorectal cancer. Dig Liver Dis Off J Ital Soc Gastroenterol Ital Assoc Study Liver 44: 66-70, 2012.

24. Gregersen LH, Jacobsen AB, Frankel LB, Wen J, Krogh A and Lund AH: MicroRNA-145 targets YES and STAT1 in colon cancer cells. PLoS One 5: e8836, 2010.

25. Valeri N, Braconi C, Gasparini P, Murgia C, Lampis A, Paulus-Hock V, Hart JR, Ueno L, Grivennikov SI, Lovat F, et al: MicroRNA-135b promotes cancer progression by acting as a downstream effector of oncogenic pathways in colon cancer. Cancer Cell 25: 469-483, 2014.

26. Ahmed FE: miRNA as markers for the diagnostic screening of colon cancer. Expert Rev Anticancer Ther 14: 463-485, 2014.

27. Saleiban A, Faxälv L, Claesson K, Jönsson JI and Osman A: miR-20b regulates expression of proteinase-activated receptor-1 (PAR-1) thrombin receptor in melanoma cells. Pigment Cell Melanoma Res 27: 431-441, 2014.

28. Lee H, Kim C, Ku JL, Kim W, Yoon SK, Kuh HJ, Lee JH, Nam SW and Lee EK: A long non-coding RNA snaR contributes to 5-fluorouracil resistance in human colon cancer cells. Mol Cells 37: 540-546, 2014

29. Qu J, Zhao L, Zhang P, Wang J, Xu N, Mi W, Jiang X, Zhang C and Qu J: MicroRNA-195 chemosensitizes colon cancer cells to the chemotherapeutic drug doxorubicin by targeting the first binding site of BCL2L2 mRNA. J Cell Physiol 230: 535-545, 2015.

30. Mussnich P, Rosa R, Bianco R, Fusco A and D'Angelo D: MiR-199a-5p and miR-375 affect colon cancer cell sensitivity to cetuximab by targeting PHLPP1. Expert Opin Ther Targets 19: 1017-1026, 2015.

31. West CM, Joseph L and Bhana S: Epidermal growth factor receptor-targeted therapy. Br J Radiol 81: S36-S44, 2008.

32. Moroni M, Veronese S, Benvenuti S, Marrapese G, SartoreBianchi A, Di Nicolantonio F, Gambacorta M, Siena S and Bardelli A: Gene copy number for epidermal growth factor receptor (EGFR) and clinical response to antiEGFR treatment in colorectal cancer: A cohort study. Lancet Oncol 6: 279-286, 2005.

33. Patel BB, Sengupta R, Qazi S, Vachhani H, Yu Y, Rishi AK and Majumdar AP: Curcumin enhances the effects of 5-fluorouracil and oxaliplatin in mediating growth inhibition of colon cancer cells by modulating EGFR and IGF-1R. Int J Cancer 122: 267-273, 2008

34. Engel J, Richters A, Getlik M, Tomassi S, Keul M, Termathe M, Lategahn J, Becker C, Mayer-Wrangowski S, Grütter C, et al: Targeting drug resistance in EGFR with covalent inhibitors: A structure-based design approach. J Med Chem 58: 6844-6863, 2015.

35. Hirao T, Nanba D, Tanaka M, Ishiguro H, Kinugasa $Y$, Doki Y, Yano M, Matsuura N, Monden M and Higashiyama S: Overexpression of ADAM9 enhances growth factor-mediated recycling of E-cadherin in human colon cancer cell line HT29 cells. Exp Cell Res 312: 331-339, 2006.

36. Hamada S, Satoh K, Fujibuchi W, Hirota M, Kanno A, Unno J, Masamune A, Kikuta K, Kume K and Shimosegawa T: MiR-126 acts as a tumor suppressor in pancreatic cancer cells via the regulation of ADAM9. Mol Cancer Res 10: 3-10, 2012. 